\title{
ON THE ORDERABILITY OF STONE-ČECH COMPACTIFICATIONS
}

\author{
S. PURISCH
}

Abstract. The Stone-Cech compactification of a Tychonoff space is orderable iff it is a pseudocompact suborderable space.

Recently, M. Venkataraman, M. Rajagopalan, and T. Soundararajan showed $[\mathbf{V}-\mathbf{R}-\mathbf{S}]$ that a necessary condition for the Stone-Cech compactification of a Tychonoff space to be orderable is that it be normal and countably compact. They also gave several sufficient conditions.

The purpose of this paper is to show that in the above theorem the condition is both necessary and sufficient if normal is changed to suborderable. This result was announced in [P2]. Also see [P1].

Recently, this result was obtained independently by $\mathrm{J}$. H. Weston.

The following proof is a shorter version of that which appeared in [P1] using a result of Gillman and Henriksen $[\mathbf{G}-\mathbf{H}]$.

Definition 1. A space $X$ is suborderable if there is a total ordering $\leqq$ on its elements such that:

(1) The original topology on $X$ is finer than its open interval topology.

(2) $X$ has a basis consisting of (possibly degenerate) intervals.

Under these conditions $\leqq$ is called an admissible order on $X$.

Definition 2. A subordered space is a pair $(X, \leqq)$ where $X$ is a suborderable space and $\leqq$ is an admissible order on $X$.

Cech, who introduced the class of suborderable spaces, proved that a space is suborderable iff it can be embedded in an (totally) orderable space.

The following definitions are equivalent to those that appear in Definition 9.3 of $[\mathbf{G}-\mathbf{H}]$.

Definition 3. Let $X$ be an ordered space, and let $\omega_{\alpha}$ be an infinite regular initial ordinal. A monotone sequence $S=\left\{x_{\xi}\right\}_{\xi<\omega_{\alpha}}$ of points in $X$ is called a $Q$ sequence if $\left\{x_{\xi}\right\}_{\xi<\lambda}$ does not converge for any nonzero limit ordinal $\lambda<\omega_{\alpha} . S$ is a nonmeasurable sequence if $\omega_{\alpha}$ is nonmeasurable.

Received by the editors February 20, 1973.

AMS (MOS) subject classifications (1970). Primary 06A05, 06A45, 54D35, 54F05.

Key words and phrases. Stone-Cech compactification, orderable space, suborderable space, nonmeasurable $Q$ sequence, Hewitt realcompactification.

(c) American Mathematical Society 1973 
As an aid to the reader referring to $[\mathbf{G}-\mathbf{H}]$ note that an ordered space has a left or right $Q$ gap of ordinality $\omega_{\alpha}$ (defined in [G-H]) iff there exists a nonconvergent $Q$ sequence of ordinality $\omega_{\alpha}$. The following lemma appears as Theorem 10.7 in $[\mathbf{G}-\mathbf{H}]$.

LEMMA 1. The Hewitt realcompactification of an ordered space $X$, whose nonconvergent $Q$ sequences are nonmeasurable, is suborderable.

Note the proof in [G-H] of Lemma 1 is also valid if $X$ were only subordered instead of ordered in both Definition 3 and Lemma 1.

LEMMA 2. A suborderable space is sequentially compact iff it is countably compact iff it is pseudocompact.

Proof. For the ordered case this lemma and its proof are part of the folklore. The same straightforward proof holds in this general setting. Also see [P1].

COROLLARY. The only $Q$ sequences of a sequentially compact subordered space $X$ are of order type $\omega_{0}$ or $\omega_{0}^{*}$. Hence, $v X$ is suborderable.

Proof. This follows directly from Definition 3, Lemma 1, and the note preceding Lemma 2.

THEOREM ([P1, P2]). The Stone- $\check{C}$ ech compactification of a Tychonoff space is orderable iff it is a pseudocompact suborderable space.

Proof. Let $X$ be a pseudocompact suborderable space. Then, $\beta X=v X$. So by Lemma 2 and the corollary $\beta X$ is suborderable and hence orderable.

Conversely, let $X$ be a Tychonoff space and let $\beta X$ be orderable. Then $X$ is suborderable. [V-R-S] shows $X$ is countably compact and hence pseudocompact.

\section{BIBLIOGRAPHY}

[G-H] L. Gillman and M. Henriksen, Concerning rings of continuous functions, Trans. Amer. Math. Soc. 77 (1954), 340-362.

[P1] S. Purisch, The categoryof ordered space, Department of Mathematics, CarnegieMellon University, Research Report 71-34, July, 1971.

[P2] — On the suborderability of metric spaces and Stone-Čech compactifications, Notices Amer. Math. Soc. 20 (1973), A-182. Abstract \#701-54-49.

[V-R-S] M. Venkataraman, M. Rajagopalan and T. Soundararajan, Orderable topological spaces, General Topology and Appl. 2 (1972), 1-10.

Department of Mathematics, Memphis State University, Memphis, Tennessee 38152

Current address: Department of Mathematics, Carnegie-Mellon University, Pittsburgh, Pennsylvania 15213 